\title{
Ruptura traqueobrônquica por trauma torácico fechado: avaliação e manuseio*
}

\author{
Wilson de Souza Stori Junior ${ }^{1}$, Marlos de Souza Coelho², José Antônio Zampier ${ }^{3}$, Gilberto Melnick ${ }^{4}$ \\ O Serviço de Cirurgia Torácica e Endoscopia Respiratória do H ospital Universitário Cajuru tratou \\ cirurgicamente dois casos de ruptura completa do brônquio principal esquerdo e um caso de ruptura \\ traqueobrônquica por trauma torácico fechado, durante um período de dois anos. A técnica utilizada para \\ reconstrução brônquica foi através de anastomose término-terminal com pontos separados utilizando-se fios \\ absorvíveis, após ressecção das margens estenosadas ou desbridamento das margens afetadas. Todos os \\ pacientes obtiveram boa evolução; o tempo médio para retirada dos drenos torácicos foi de 3,6 dias e o \\ tempo médio para alta do Serviço de Cirurgia Torácica foi de 5,6 dias. 0 seguimento dos pacientes foi feito \\ através de radiografia simples de tórax em duas incidências (PA e perfil), provas de função pulmonar e \\ broncofibroscopia. (J Pneumol 1999;25(2):118-123)
}

\section{Tracheobronchial rupture by blunt chest trauma: evaluation and management}

Two cases of complete bronchial rupture and one case of tracheobronchial rupture by blunt chest trauma were treated at the Thoracic Surgical and Respiratory Endoscopy Department of the Hospital Universitário Cajuru in a period of 2 years. The surgical technique used was end-to-end anastomosis with interrupt absorbable suture after either resection of the stenotic edges or debridement of the lacerated edges. All three patients have had good recovery. The mean period to remove the chest tubes was 3.6 days, and the mean period of discharge from the Thoracic Surgical Department was 5.6 days. The foll ow-up was made using chest X-ray (PA and lateral projections), lung function tests, and bronchoscopy.

Siglas e abreviaturas utilizadas neste trabal ho

CV - Capacidade vital

$V_{E F}$ - Volume expiratório forçado no primeiro segundo

PA - Póstero-anterior

TAC - Tomografia axial computadorizada

PO - Pós-operatório

LC2 - Carina entre o brônquio segmentar da língula do lobo superior esquerdo e o brônquio lobar inferior esquerdo

$\mathrm{SO}_{2}$ - Saturação de oxigênio

bpm - Batimentos por minuto

ipm - Incursões por minuto

* Trabalho realizado pelo Departamento de Cirurgia Torácica e Endoscopia Respiratória do Hospital U niversitário Cajuru-PUC-PR.

1. Residente do 3 ano de Cirurgia Torácica.

2. Chefe do Serviço de Cirurgia Torácica e Endoscopia Respiratória.

3. Médico Assistente do Serviço de Cirurgia Torácica e Endoscopia Respiratória.

4. Aluno do 60 ano de Medicina da UFPR e Ex-Voluntário do Serviço de Cirurgia Torácica e Endoscopia Respiratória.

Endereço para correspondência - Wilson de Souza Stori J unior, Av. Visconde de Guarapuava, 4.905, Apto. 301, Batel - 80240-010 Curitiba, PR. e-mail: stori@cwb.matrix.com.br

Recebido para publicação em 20/11/98. Reapresentado em 4/3/99. Aprovado, após revisão, em 10/3/99.

Descritores - Ruptura traqueobrônquica. Trauma torácico fechado. A valiação. Tratamento.

Key words - Tracheobronchial rupture. Blunt chest trauma. Evaluation. Treatment.

\section{INTRODUÇÃO}

Embora haja melhora no reconhecimento, lesões brônquicas continuam raras em vítimas de grandes traumas ${ }^{(1)}$. A incidência de trauma não penetrante vem crescendo significativamente neste século devido ao aumento da velocidade dos meios de transporte ${ }^{(2)}$. Nos últimos anos, progressos foram feitos no reconhecimento e tratamento de lesões de vias aéreas $^{(1)}$.

A grande maioria das lesões traqueobrônquicas ocorre como resultado de colisões automobilísticas. A pessoa que mais freqüentemente está envolvida é o motorista, embora os outros passageiros também possam sofrer tais lesões, incluindo as crianças $^{(3)}$. Os mecanismos de lesão da árvore traqueobrônquica no trauma torácico fechado podem ser: 1) aumento abrupto na pressão intratraqueal e/ ou intrabrônquica com a glote fechada, levando a laceração longitudinal na parede membranosa da traquéia; 2) desaceleração brus$\mathrm{ca}$, produzindo forças de cisalhamento, fazendo com que 
ocorra ruptura da traquéia em um ponto de fixação (carina e cricóide); 3) o esmagamento de tórax faz com que haja diminuição do diâmetro ântero-posterior da cavidade torácica, levando a tração lateral dos pulmões, produzindo laceração ou ruptura da traquéia e/ ou brônquios próxima da carina; 4) deslocamento do coração para trás, expondo a carina a torção $0^{(1,3-5)}$.

\section{OBJETIVO}

A valiar o diagnóstico e a eficácia da cirurgia para reconstrução da traquéia e do brônquio e sua restauração funcional através de radiografia de tórax, broncofibroscopia e provas de função pulmonar.

\section{Materiais e mÉTOdos}

Durante o período de $1 / 6 / 96$ a $1 / 6 / 98$, foram analisados, prospectivamente, três pacientes vítimas de trauma torácico fechado. Dois pacientes apresentavam ruptura completa de brônquio principal esquerdo e um, ruptura traqueobrônquica. Os pacientes foram atendidos e conduzidos pelo Serviço de Cirurgia Torácica e Endoscopia Respiratória do Hospital Universitário Cajuru-PUC-PR; um paciente (caso 2) foi inicialmente atendido no interior do Estado e posteriormente encaminhado e outro (caso 1 ) foi atendido por outro serviço em nosso Hospital e posteriormente foi solicitada nossa avaliação. Dois pacientes eram do sexo feminino e um, do masculino. A idade média foi de 22,6 anos.

\section{Caso 1}

Paciente do sexo feminino, 22 anos de idade, politraumatizada, vítima de atropelamento, foi admitida no Hospital Universitário Cajuru em estado torporoso. A presentava pressão arterial de $130 / 80 \mathrm{mmH}$, pulso de $120 \mathrm{bpm}$ e taquipnéia de 30ipm. Ao exame físico do tórax foi encontrado murmúrio vesicular diminuído à esquerda. 0 estudo radiológico de tórax mostrou fratura de $2 \stackrel{0}{a}$ arco costal à esquerda e de 30 e 4ำ arcos costais à direita, pneumotórax de médio volume à esquerda e desvio das estruturas do mediastino para a direita. Possuía as seguintes lesões associadas ao trauma torácico: traumatismo cranioencefálico, com TAC normal, ferida cortocontusa em região supra-orbital, fratura do úmero e olécrano à esquerda.

Como conduta imediata foi realizada drenagem torácica fechada sob selo d'água à esquerda. Durante a evolução, o débito de sangue encontrado no dreno de tórax diminuiu de $350 \mathrm{ml}$ no primeiro dia para $150 \mathrm{ml}$ no segundo dia. Na evolução radiológica do tórax a paciente apresentou pneumotórax de médio volume no primeiro dia após a drenagem fechada, evoluindo com expansão total no controle radiológico do 3 o dia. Sem sinais de complicações clínico-radiológicas, o dreno torácico foi retirado ao final do 30 dia. Ao término do tratamento ortopédico a paciente recebeu alta hospitalar.
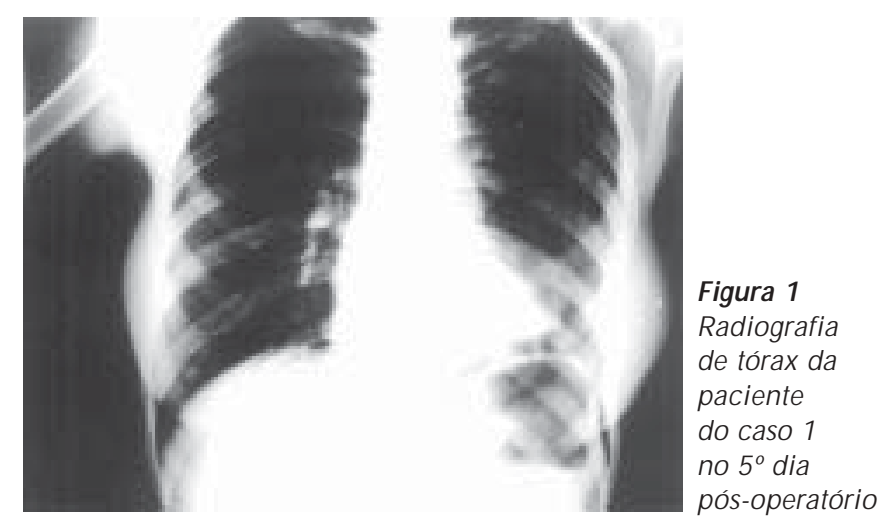

Dois meses após a retirada do dreno torácico, a paciente retornou ao Serviço de Cirurgia Torácica do Hospital Universitário Cajuru apresentando dispnéia aos pequenos esforços, tosse com expectoração amarelada, dor em hemitórax esquerdo do tipo contínuo e de moderada intensidade e murmúrio vesicular abolido no hemitórax esquerdo. A radiografia de tórax mostrou elevação da hemicúpula diafragmática esquerda, desvio da traquéia e das estruturas do mediastino para o mesmo lado, retração dos espaços intercostais e velamento completo do hemitórax esquerdo.

O estudo tomográfico do tórax mostrou, além das lesões já identificadas na radiografia de tórax, diminuição gradativa da luz do brônquio principal esquerdo.

A paciente foi submetida a endoscopia respiratória, que mostrou o brônquio principal esquerdo terminando em fundo cego a cerca de $1,5 \mathrm{~cm}$ da carina traqueal com pouca quantidade de secreção mucosa. Com o diagnóstico de ruptura brônquica comprovado, o tratamento cirúrgico foi indicado.

0 acesso para a broncoplastia foi através de uma toracotomia póstero-lateral esquerda no 50 espaço intercostal. Com a dissecção da janela aortopulmonar, os cotos brônquicos proximal e distal lesados foram identificados e ambos terminavam em fundo cego. A pós a ressecção dos segmentos brônquicos comprometidos, foi realizada anastomose términoterminal com pontos separados utilizando fio absorvível vicryl 2-0 e inseridos dois drenos torácicos.

No 3 o dia Po não havia fuga aérea pelo dreno torácico, porém, radiologicamente, a expansão completa do pulmão foi evidenciada somente no 5o dia Po (fig. 1). Os drenos torácicos foram retirados nesse mesmo dia e a paciente recebeu alta hospitalar no 60 dia após a cirurgia.

A paciente foi seguida ambulatorialmente com provas de função pulmonar, radiografia de tórax e broncofibroscopia. $\mathrm{Na}$ prova de função pulmonar do 3 o mês de pós-operatório foi encontrada CV de 1,95 (55\%) e $\mathrm{VEF}_{1}$ de 1,60 (55\%). No 4 o mês de pós-operatório a CV era de 2,28 (65\%) e o $\mathrm{VEF}_{1}$ de $2,12(69,3 \%)$. A broncofibroscopia realizada no 3 을 e no 4 은 mês após a cirurgia identificou diminuição do calibre do brônquio principal esquerdo de aproximadamente $30 \%$ da luz, 


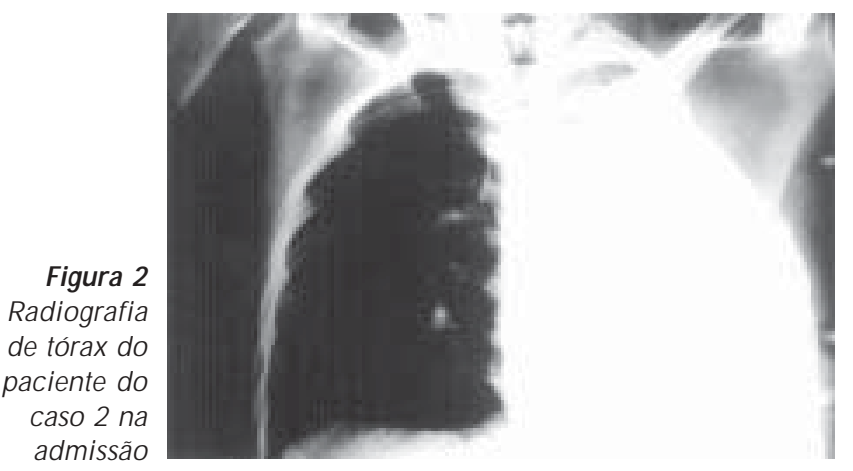

próximo à LC2 (linha de sutura). O s brônquios segmentares e subsegmentares não mostraram alterações. A radiografia de tórax mostrou os pulmões completamente expandidos e 0 mediastino em posição normal.

\section{Caso 2}

Paciente do sexo masculino, 25 anos, vítima de acidente de trabalho, quando uma tora de madeira caiu de um caminhão sobre seu dorso, sendo atendido inicialmente em um hospital no interior do Estado, onde foi submetido a toracotomia esquerda e não foi encontrada lesão. Q uinze dias após o acidente o paciente foi transferido para o Serviço de Cirurgia Torácica e Endoscopia Respiratória do Hospital Universitário Cajuru. A radiografia de tórax realizada no hospital de origem mostrou luxação esternoclavicular esquerda, luxação costovertebral do 10 arco costal esquerdo, fratura escalonada do 20 ao 5 ㅇ arco costal posterior à esquerda e pneumomediastino.

$\mathrm{Na}$ admissão apresentava intensa dispnéia, frêmito toracovocal diminuído, murmúrio vesicular ausente, drenagem torácica fechada à esquerda. A radiografia de tórax mostrou, além das alterações observadas na radiografia anterior, desvio do mediastino para a esquerda, velamento completo do hemitórax esquerdo e retração dos espaços intercostais desse mesmo lado (fig. 2). A tomografia (fig. 3) evidenciou total opacificação e diminuição volumétrica do hemitórax esquerdo, brônquio principal esquerdo visível no terço proximal com afilamento gradativo e obstrução total no terço distal, colapso total do pulmão esquerdo e desvio do mediastino para a esquerda, compatível com fratura de brônquio principal esquerdo e condensação pulmonar no lobo inferior direito, compatível com pneumonia. A broncofibroscopia demonstrou o brônquio principal esquerdo terminando em fundo cego a $2 \mathrm{~cm}$ da carina traqueal.

A presentava as seguintes lesões associadas ao trauma torácico: fratura do úmero direito e lesão do plexo braquial esquerdo.

Confirmado o diagnóstico de ruptura do brônquio principal esquerdo, a broncoplastia foi indicada. Realizada toracotomia póstero-lateral esquerda ao nível do 5 o espaço intercostal. A pós a identificação e desbridamento dos cotos brônquicos proximal e distal, foi confeccionada uma anastomose

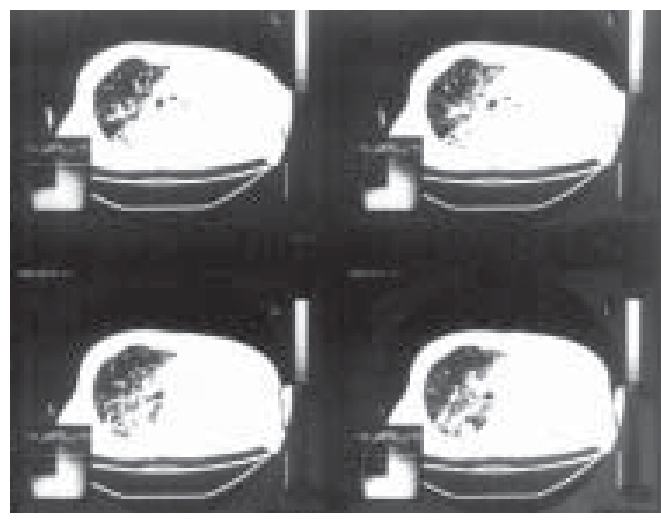

Figura 3 Tomografia computadorizada do paciente do caso 2 na admissão
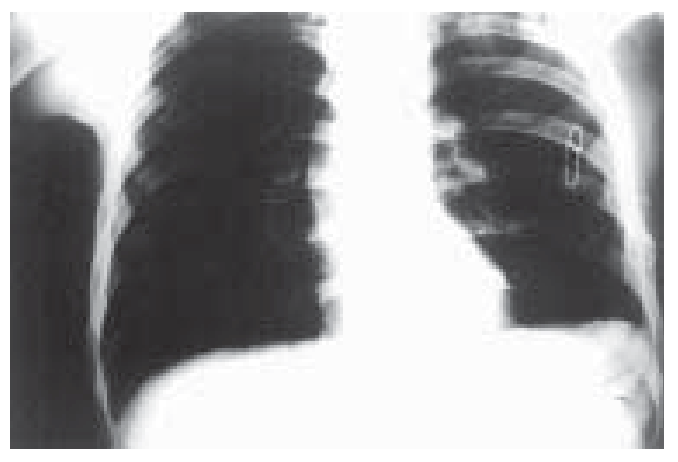

Figura 4

Radiografia de tórax do paciente do caso 2 no 8 o mês pós-operatório

término-terminal do brônquio principal esquerdo com pontos separados utilizando-se fio absorvível vicryl 3-0.

o paciente teve boa evolução clínica e apresentou, em radiografia de controle, expansão completa do pulmão esquerdo, sendo então retirados os drenos torácicos no 30 dia de pós-operatório. Recebeu alta do Serviço de Cirurgia Torácica no 50 dia de pós-operatório.

Sua evolução foi acompanhada com provas de função pulmonar, estudo radiológico simples de tórax e broncofibroscopia. Na prova de função pulmonar do 30 mês de pós-

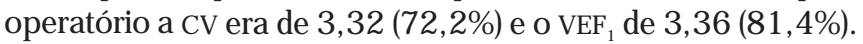

A radiografia de tórax do 80 mês de pós-operatório mostrou pulmão esquerdo completamente expandido (fig. 4).

A broncofibroscopia realizada no 90 dia e no 8 o mês de pós-operatório revelou diminuição do calibre do brônquio próximo à LC2 (a $2 \mathrm{~cm}$ da carina traqueal), de aproximadamente $20 \%$ da luz, no local da anastomose.

\section{Caso 3}

Paciente feminina, 21 anos, vítima de acidente automobilístico sem cinto de segurança, foi admitida no Pronto-Socorro do H ospital U niversitário Cajuru apresentando trauma torácico de grande intensidade. A o exame físico apresentava-se com movimento paradoxal em região anterior do hemitórax direito, enfisema subcutâneo envolvendo o tórax e o pescoço, intensa dispnéia e ferida em mento de aproximadamente $2 \mathrm{~cm}$. Sinais vitais encontrados na sala de emergência: $\mathrm{SO}_{2}$ de 99\%; pressão arterial de 120/80mmHg; pulso de $120 \mathrm{bpm}$; freqüência respiratória de $44 \mathrm{ipm}$. 

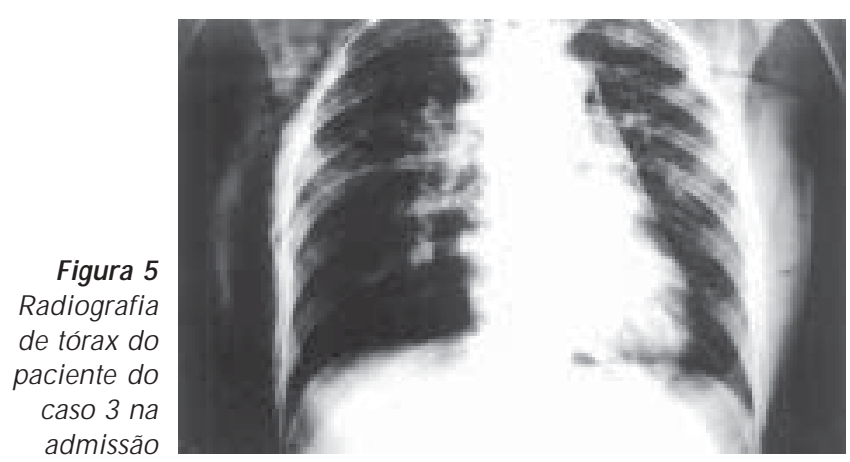

O estudo radiológico inicial do tórax mostrou enfisema subcutâneo à direita e discreto pneumomediastino; não foram encontradas fraturas de costelas e pneumotórax (fig. 5). 0 estudo radiológico de tórax foi repetido horas depois e identificou-se pneumotórax de grande volume à direita (fig. 6). A paciente foi submetida à drenagem torácica fechada em selo d'água e broncofibroscopia, que identificou uma lesão de aproximadamente $1 \mathrm{~cm}$ na parede ântero-lateral esquerda no terço inferior da traquéia e outra lesão na traquéia que se iniciava $2 \mathrm{~cm}$ acima da carina traqueal na parede póstero-lateral direita, na transição da cartilagem com a parede membranosa e estendia-se através do brônquio principal direito, terminando junto ao óstio do brônquio lobar superior.

A paciente foi levada imediatamente ao centro cirúrgico e submetida à cirurgia para reconstrução brônquica. Foi realizada toracotomia póstero-lateral direita no 4 ㅇ espaço intercostal. A pós a ligadura da veia ázigos e abertura da pleura mediastinal, foi identificada uma lesão transversal na parede ântero-lateral esquerda com $1 \mathrm{~cm}$ de extensão, e outra longitudinal na parede póstero-lateral direita (transição entre a parede cartilaginosa e a membranosa), que se iniciava $2 \mathrm{~cm}$ acima da carina traqueal e estendia-se até o brônquio lobar superior direito, com extensão de aproximadamente $4,5 \mathrm{~cm}$. Foi efetuado o desbridamento das bordas das lesões e realizada sutura com pontos separados utilizando-se fio absorvível vicryl 3-0. A área de sutura foi recoberta com pleura parietal.

No controle radiológico do primeiro dia de pós-operatório, evidenciava-se pneumotórax de pequeno volume em campo inferior; no segundo dia o pulmão estava completamente expandido. Os drenos de tórax foram retirados no 30 dia de pós-operatório e a paciente recebeu alta hospitalar no 60 dia.

A paciente recebeu seguimento ambulatorial com provas de função pulmonar, radiografia de tórax e broncofibroscopia. Na prova de função pulmonar do 3 o mês de pós-operatório a CV foi de 3,68 $(98,8 \%)$ e o $\mathrm{VEF}_{1}$ de $2,76(85,9 \%)$.

A broncofibroscopia realizada no 30 mês após a alta hospitalar (fig. 7) identificou granuloma com $5 \mathrm{~mm}$ de diâmetro no terço inferior da traquéia e a luz do brônquio principal direito ligeiramente diminuída de calibre em relação à do esquerdo. Não foi identificada qualquer alteração no brônquio
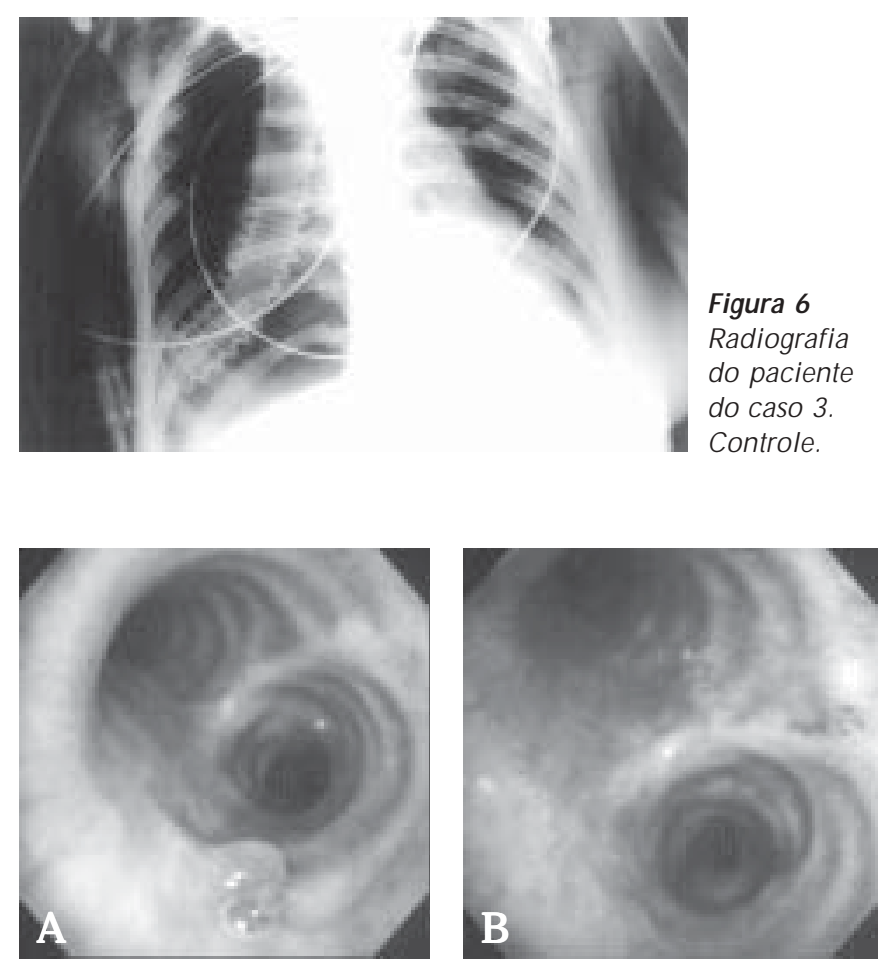

Figura 7 - Broncoscopia do paciente do caso 3 no 30 mês de pósoperatório. A) Granuloma de $5 \mathrm{~mm}$ na parede brônquica. B) Discreta diminuição de calibre da luz do brônquio principal direito.

intermediário, bem como no brônquio lobar superior, inferior e médio.

A radiografia de tórax mostrou os pulmões completamente expandidos, sem qualquer alteração radiológica.

\section{DISCUSSÃO}

A primeira reparação cirúrgica de uma ruptura brônquica causada por trauma torácico fechado foi relatada em 1947 por Kinsella e J ohnsrud(6). Em 1959, H ood e Sloan ${ }^{(7)}$ relataram melhora importante nos resultados após reparação cirúrgica imediata dessas lesões. Desde então, várias séries demonstraram que o diagnóstico precoce e o tratamento cirúrgico dessas lesões diminuem as complicações e melhoram os resultados a longo prazo ${ }^{(8,9)}$.

Pacientes que possuem solução de continuidade da pleura mediastinal apresentam-se com dispnéia; evidência de pneumotórax, ocasionalmente hipertensivo; enfisema mediastinal e subcutâneo; e mínima hemoptise. Se realizada drenagem sob selo d'água, haverá importante fuga aérea e geralmente o pulmão não se expande. Esses pacientes podem apresentar insuficiência respiratória grave ${ }^{(3)}$.

Se não houver lesão da pleura mediastinal, surpreendentemente haverá poucos sinais e sintomas. A ruptura brônquica na maioria das vezes é completa e geralmente não há pneumotórax. Se houver pneumotórax, ele não será grande e a expansão do pulmão ocorrerá normalmente, sem fuga aérea persistente ${ }^{(3)}$. 
Pode ocorrer atelectasia em intervalo entre 6 e 21 dias. A lguns pacientes não apresentam manifestações clínicas significantes e recebem alta hospitalar sem o diagnóstico. 0 diagnóstico só será suspeito em radiografia de controle ou se o paciente apresentar sintomas respiratórios, podendo permanecer indetectável por semanas, meses ou anos ${ }^{(3)}$.

0 enfisema subcutâneo e a dispnéia são os sinais iniciais mais comuns. Recentemente, em uma série de 13 pacientes com ruptura traqueobrônquica por trauma torácico fechado, $85 \%$ dos pacientes tinham enfisema subcutâneo e 77\% apresentavam dispnéia( ${ }^{(3,4,10)}$. Em nossa casuística, somente um paciente apresentou enfisema subcutâneo, porém a dispnéia foi um sintoma precoce em todos os casos. No estudo radiológico tardio foi observada atelectasia em dois casos. Nenhum paciente apresentou hemoptise e sinal de Hamman. O estudo radiológico inicial de tórax mostrou pneumotórax em somente um dos três casos, porém em outro a radiografia de tórax foi repetida pouco tempo depois e mostrou um pneumotórax de grande volume. 0 pneumomediastino esteve presente em dois pacientes da série. Baumgartner et al. . $^{(4)}$ encontraram pneumotórax em 6 de 9 pacientes e pneumomediastino em 3 de 9 pacientes. J ones et al.(10) relataram que o pneumotórax esteve presente em 5 de 13 casos e 0 pneumomediastino em 6 de 13 casos. Outros achados radiográficos no tórax, presentes em nossa série, incluíam fraturas de arcos costais (2 em 3 pacientes), desvio do mediastino (1 em 3 pacientes) e luxação esternoclavicular e costovertebral (1 em 3 pacientes).

Devido aos mecanismos de lesão, que exigem grande quantidade de energia cinética, devemos esperar que haja transferência desta energia cinética, produzindo lesões associadas ${ }^{(3,4)}$. Baumgartner et al..$^{(4)}$ encontraram ruptura traqueobrônquica isolada em somente 2 entre 9 . E em outra grande série foram encontradas 48 lesões associadas em 13 pacientes ${ }^{(10)}$.

Oitenta por cento das rupturas brônquicas ocorrem dentro de distância de até $2,5 \mathrm{~cm}$ da carina. As lesões brônquicas são igualmente distribuídas entre os brônquios principais direito e esquerdo ${ }^{(6,11)}$. Rupturas traqueobrônquicas por trauma torácico fechado geralmente são únicas e transversais, mas também podem ser longitudinais ou complexas. São consideradas complexas quando a ruptura possui mais de uma forma ou ocorre em mais de um local(12).

Em revisão de artigos publicados na língua inglesa pelo período de 1970 a 1990, Symbas et al. (1992) encontraram 183 casos de ruptura da via aérea, sendo que $136(74 \%)$ eram transversais, $33(18 \%)$ longitudinais e $14(8 \%)$ complexas. Das 136 lesões transversais, 7 rupturas (4\%) ocorreram na traquéia cervical, 22 (12\%) na traquéia torácica, 47 (25\%) no brônquio principal direito, 31 (17\%) no brônquio principal esquerdo e 29 (16\%) em brônquio lobar. Das 33 lacerações longitudinais, 12 (6,5\%) ocorreram na traquéia cervical, $18(10 \%)$ na traquéia torácica, 2 (1\%) no brônquio principal direito e $1(0,5 \%)$ no brônquio principal esquerdo. As rupturas complexas, nos 14 casos, envolveram mais freqüente- mente ou a traquéia e o brônquio principal direito ou a traquéia e ambos os brônquios principais, em diferentes combinações ${ }^{(12)}$.

A incidência de ruptura traqueobrônquica em vítimas de trauma torácico fechado é rara, apesar da melhora no reconhecimento dessas lesões. Bertelsen e H owitz, encontraram 33 lesões traqueobrônquicas em 1.178 pacientes $(2,8 \%)$ que sofreram trauma torácico fechado(13). Iwasaki et al., em 1994, relataram incidência de $2,1 \%$ de lesões traqueobrônquicas em vítimas de trauma torácico fechado(14). Dougall et al. relatam incidência de lesões da árvore traqueobrônquica em pacientes que sofreram trauma torácico fechado de aproximadamente $1 \%{ }^{(15)}$. Coelho et al. encontraram, em recente análise de 643 pacientes com trauma torácico fechado, 2 $(0,31 \%)$ com ruptura completa do brônquio principal esquerdo ${ }^{(16)}$.

Para o diagnóstico é necessário que haja, principalmente, elevado índice de suspeita. Esse fato, entretanto, não pode ser muito valorizado, porque mais de dois terços dos pacientes possuem sinais vitais estáveis no primeiro exame. Desse modo, devem-se procurar outros sinais, que podem ser sutis e de difícil reconhecimento. Tais sinais podem ser encontrados de forma resumida no quadro $1^{(5)}$.

Os exames diagnósticos incluem radiografia de tórax e endoscopia respiratória. A radiografia simples de tórax é freqüentemente útil e geralmente é o primeiro exame disponível. Fraturas de costelas, especialmente da $1 \underline{a}$ a $5 \underline{a}$, fraturas de outros ossos do tórax, pneumotórax, pneumomediastino, pneumopericárdio e enfisema subcutâneo, são alterações sugestivas de lesão da árvore traqueobrônquica e facilmente detectáveis na radiografia simples do tórax. Entretanto, aproximadamente um quarto dos pacientes com lesão traqueobrônquica tem radiografias iniciais do tórax completamente normais ${ }^{(5)}$. Um sinal radiográfico raro, porém considerado altamente específico de ruptura completa de brônquios principais, é o sinal do "pulmão caído". Este achado radiográfico consiste no deslocamento inferior do pulmão, distante do mediastino, e sem estruturas hilares visíveis ${ }^{(17)}$.

0 exame de escolha é a broncoscopia, que pode ser rígida ou flexível ${ }^{(3,5)}$. As indicações iniciais para broncoscopia são: hemoptise, enfisema subcutâneo, fuga aérea sem melhora, pneumotórax persistente e pneumomediastino(5). A lesão brônquica pode não ser identificada no primeiro procedimento endoscópico em aproximadamente $50 \%$ dos pacientes ${ }^{(3)}$. No

\section{QUADRO 1}

Achados na lesão da árvore traqueobrônquica( ${ }^{(5)}$

Tosse

Dispnéia

Hemoptise

Enfisema subcutâneo

Fratura de ossos do tórax (costela, escápula, clavícula e esterno) Pneumotórax

Pneumomediastino

Pneumopericárdio 
exame realizado logo após o trauma, os achados podem incluir ruptura completa ou parcial da traquéia, laceração traqueal, ruptura completa ou parcial do brônquio, laceração brônquica, ou ainda pode apresentar apenas sinal que sugere a presença de lesão traqueal e/ ou brônquica, tal como sangramento. No exame realizado tardiamente, podem ser encontrados sinais indiretos de ruptura brônquica, tais como tecido de granulação; estenose parcial do brônquio, quando a ruptura foi parcial; ou o brônquio terminando em fundo cego, com a superfície recoberta por mucosa normal, quando a ruptura foi completa ${ }^{(3,18)}$.

Lesões pequenas na traquéia e com saída de ar limitada podem ser conduzidas de forma conservadora, uma vez afastadas lesões associadas, como, por exemplo, uma lesão no esôfago(3).

$\mathrm{Na}$ maioria dos serviços, assim como o nosso, o acesso utilizado para o terço proximal e médio da traquéia é uma

\section{REFERÊNCIAS}

1. Shields TW. General thoracic surgery. $4^{\text {th }}$ ed. Philadelphia: Williams \& Wilkins, 1994;1:767-783.

2. Lin MY, Wu MH, Chan CS, Lai WW, Chou NS, Tseng YL. Bronchial rupture caused by blunt chest injury. Ann Emerg Med 1995;25:412 415.

3. Hood RM, Boyd AD, Culliford AT. Thoracic trauma. $1^{\text {st }}$ ed. Philadelphia: W.B. Saunders, 1989;245-266.

4. Baumgartner F, Sheppard B, Virgilio C, Esrig B, Harrier D, Nelson RJ , Robertson J M. Tracheal and main bronchial disruptions after blunt chest trauma: presentation and management. Ann Thorac Surg 1990; 50:569-574.

5. Blaisdell FW, Trunkey DD. Cervicothoracic trauma. $2^{\text {nd }}$ ed. New YorkStuttgart: Thieme Medical Publishers, 1994;178-189.

6. Kinsella TJ , J ohnsrud LW. Traumatic rupture of the bronchus. J Thorac Surg 1947;16:571-583.

7. Hood RM, Sloan HE. Injuries of the trachea and major bronchi. J Thorac Cardiovasc Surg 1959;38:458-480.

8. Kirsh MM, Orringer MB, Behrendt DM, et al. Management of tracheobronchial disruption secondary to nonpenetrating trauma. Ann Thorac Surg 1976;22:93-101.

9. Ecker RR, Libertini RV, Rea WJ, et al. Injuries of the trachea and bronchi. Ann Thorac Surg 1971;11:289-298. incisão cervical em colar, podendo ser combinada com esternotomia parcial se for necessário. Para lesões da traquéia distal, da carina, dos brônquios à direita e para o terço proximal do brônquio principal esquerdo, o acesso de escolha é a toracotomia direita; porém para lesões do terço médio e distal do brônquio principal esquerdo, o acesso de escolha é a toracotomia esquerda. A anastomose é feita com pontos separados utilizando-se fios absorvíveis e, sempre que possível, os pacientes são extubados ainda no centro cirúrgico. A traqueostomia deve ser feita somente se houver necessidade de controlar secreções ou se houver necessidade de manter o paciente em ventilação mecânica por mais de 4 ou 5 dias. Também não há necessidade de drenagem da ferida cirúrgica na ausência de lesão esofágica.

Em alguns casos, devido à complexidade das rupturas, pode ser necessária circulação extracorpórea para assegurar adequada reparação das lesões ${ }^{(12)}$.

10. J ones WS, Mavroudis C, Richardson J D, et al. Management of tracheobronchial disruption resulting from blunt trauma. Surgery 1984;95: 319-322.

11. Hartley C, Morritt GN. Bronchial rupture secondary to blunt chest trauma. Thorax 1993;48:183-184.

12. Symbas PN, J usticz AG, Ricketts RR. Rupture of the airways from blunt trauma: treatment of complex injuries. Ann Thorac Surg 1992; 54:177-183.

13. Bertelsen S, Howitz P. Injuries of the trachea and bronchi. Thorax 1972;27:188.

14. Iwasaki M, Kaga K, O gawa J, Inoue H, Shohtsu A. Bronchoscopy findings and early treatment of patients with blunt tracheo-bronchial trauma. J Cardiovasc Surg 1994;35:269-271.

15. Dougall AM, Paul ME, Finely RJ, et al. Chest trauma - Current morbidity and mortality. J Trauma 1977; 17:547-553.

16. Coelho MS, Lira EJT, Stori WSJ , Zanin SA, Zampier J A. Traumatismo torácico: análise de 1.554 casos. J Pneumol 1998;24(Supp 1): S42.

17. Endress C, Guyot RD, Engels J A. The "fallen lung with absent hilum" signs of complete bronchial transection. Ann Emerg Med 1991;20: 317-318.

18. Prakash UBS. Bronchoscopy. New York: Raven Press, 1994;81-88. 